Special Issue: 25 years of the Mizar Mathematical Library

FORMALIZED MATHEMATICS

Vol. 22, No. 2, Pages $177-178,2014$

DE GRUYTER

DOI: $10.2478 /$ forma-2014-0018

\title{
A Note on the Seven Bridges of Königsberg Problem
}

\author{
Adam Naumowicz \\ Institute of Informatics \\ University of Białystok \\ Sosnowa 64, 15-887 Białystok \\ Poland
}

\begin{abstract}
Summary. In this paper we account for the formalization of the seven bridges of Königsberg puzzle. The problem originally posed and solved by Euler in 1735 is historically notable for having laid the foundations of graph theory, cf. [7. Our formalization utilizes a simple set-theoretical graph representation with four distinct sets for the graph's vertices and another seven sets that represent the edges (bridges). The work appends the article by Nakamura and Rudnicki 10] by introducing the classic example of a graph that does not contain an Eulerian path.

This theorem is item \#54 from the "Formalizing 100 Theorems" list maintained by Freek Wiedijk at http://www.cs.ru.nl/F.Wiedijk/100/.
\end{abstract}

MSC: 05C45 05C62 03B 35

Keywords: Eulerian paths; Eulerian cycles; Königsberg bridges problem

MML identifier: GRAPH_3A, version: $8.1 .03 \quad 5.23 .1213$

The notation and terminology used in this paper have been introduced in the following articles: [11], [2], 8], [3], [4], [9, [10], 6], [1], [13, [12], and [5].

The functors: KVertices and KEdges yielding sets are defined by terms,

(Def. 1) $\{0,1,2,3\}$,

(Def. 2) $\{10,20,30,40,50,60,70\}$,

respectively. The functors: KSource and KTarget yielding functions from KEdges into KVertices are defined by terms,

(Def. 3) $\{\langle 10,0\rangle,\langle 20,0\rangle,\langle 30,0\rangle,\langle 40,1\rangle,\langle 50,1\rangle,\langle 60,2\rangle,\langle 70,2\rangle\}$,

(Def. 4$) \quad\{\langle 10,1\rangle,\langle 20,2\rangle,\langle 30,3\rangle,\langle 40,2\rangle,\langle 50,2\rangle,\langle 60,3\rangle,\langle 70,3\rangle\}$, respectively. The functor KönigsbergBridges yielding a graph is defined by the term 
(Def. 5) 〈KVertices, KEdges, KSource, KTarget $\rangle$.

Let us observe that KönigsbergBridges is finite and connected.

Let us consider a vertex $v$ of KönigsbergBridges. Now we state the propositions:

(1) If $v=0$, then the degree of $v=3$. Proof: EdgesIn $v=\emptyset$ by [3, (1)]. EdgesOut $v=\{10,20,30\}$ by [3, (1)]. The degree of $v=3$ by [10, (24)].

(2) If $v=1$, then the degree of $v=3$. Proof: EdgesIn $v=\{10\}$ by [3, (1)]. EdgesOut $v=\{40,50\}$ by [3, (1)]. The degree of $v=3$ by [10, (24)].

(3) If $v=2$, then the degree of $v=5$. Proof: EdgesIn $v=\{20,40,50\}$ by [3, (1)]. EdgesOut $v=\{60,70\}$ by [3, (1)]. The degree of $v=5$ by [10, (24)].

(4) If $v=3$, then the degree of $v=3$. Proof: EdgesIn $v=\{30,60,70\}$ by [3, (1)]. EdgesOut $v=\emptyset$ by [3, (1)]. The degree of $v=3$ by [10, (24)].

Now we state the propositions:

(5) Seven Bridges of Königsberg:

There exists no path $p$ of KönigsbergBridges such that $p$ is cyclic and Eulerian. The theorem is a consequence of (1).

(6) There exists no path $p$ of KönigsbergBridges such that $p$ is non cyclic and Eulerian. The theorem is a consequence of (4), (1), and (2).

\section{REFERENCES}

[1] Grzegorz Bancerek. The ordinal numbers, Formalized Mathematics, 1(1):91-96, 1990.

[2] Grzegorz Bancerek and Krzysztof Hryniewiecki. Segments of natural numbers and finite sequences. Formalized Mathematics, 1(1):107-114, 1990.

[3] Czesław Byliński. Functions and their basic properties Formalized Mathematics, 1(1): 55-65, 1990.

[4] Czesław Byliński. Functions from a set to a set Formalized Mathematics, 1(1):153-164, 1990.

[5] Czesław Byliński. Some basic properties of sets Formalized Mathematics, 1(1):47-53, 1990.

[6] Czesław Byliński and Piotr Rudnicki. The correspondence between monotonic many sorted signatures and well-founded graphs. Part 1. Formalızed Mathematıcs, 5(4):577$582,1996$.

[7] Gary Chartrand. Introductory Graph Theory. New York: Dover, 1985.

[8] Agata Darmochwał. Finite sets. Formalized Mathematics, 1(1):165-167, 1990.

[9] Krzysztof Hryniewiecki. Graphs. Formalized Mathematics, 2(3):365-370, 1991.

[10] Yatsuka Nakamura and Piotr Rudnicki. Euler circuits and paths. Formalized Mathematics, 6(3):417-425, 1997.

[11] Andrzej Trybulec. Enumerated sets. Formalized Mathematics, 1(1):25-34, 1990.

[12] Zinaida Trybulec. Properties of subsets Formalized Mathematics, 1(1):67-71, 1990.

[13] Edmund Woronowicz. Relations and their basic properties. Formalized Mathematics, 1 (1):73-83, 1990. 